\title{
High recycling outer divertor regimes after type-I ELMs at high density in ASDEX Upgrade
}

\author{
M. Wischmeier*, A. Kallenbach, A. V. Chankin, D. P. Coster, \\ T. Eich, A. Herrmann, H. W. Müller and \\ ASDEX Upgrade team \\ Max-Planck-Institut für Plasmaphysik, EURATOM-Association, Boltzmannstr. 2, \\ D-85748, Garching, Germany
}

\begin{abstract}
Experiments have been conducted in SNL type-I ELMy H-mode on ASDEX Upgrade. Depending on the upstream conditions in the Scrape-off-layer and core plasma, different temporal evolutions of the plasma regime in the outer divertor are identified during the recovery period following an ELM. The divertor plasma is characterized using experimental measurements such as line radiation $\left(D_{\alpha}, C I I I\right)$, spatially resolved thermography (IR) and fixed Langmuir probes (LP). Whilst in one case a first clear temporal peak in line radiation $\left(D_{\alpha}, C I I I\right)$ as well as power (IR) and particle fluxes (LP) onto the outer divertor plate is followed by a quiescent interELM phase, in the other case this initial peak is followed by a broader temporal peak in all but the IR and CIII signals. A possible explanation based on simple analytic considerations is presented.
\end{abstract}

Key words: 
PSI-17 keywords: ASDEX-Upgrade, ELM, Divertor, Edge Modelling

JNM keywords: Plasma Properties

PACS: 52.55.Fa, 52.65.-y, 52.25.-b

\section{Introduction}

A future experimental device such as ITER is expected to operate in the high confinement mode, H-mode. In H-mode edge localized modes, ELMs [1], an MHD instability localized at the edge of the plasma, are regularly observed and release a fraction of confined plasma energy and particles into the scrapeoff layer, SOL. This expulsion is a transient phenomenon on a time scale below $1 m s$. Most of the released particles and energy during an ELM cross the separatrix around the outer midplane. Each ELM causes increased transient power and particle loads to the divertor targets and the main chamber walls, MCW. A high recycling divertor can be assessed at low $\bar{n}_{e}$ and the partially detached outer divertor needed for increasing the lifetime of the outer divertor in ITER [2], at increased $\bar{n}_{e}$. The contribution presented here treats a high recycling outer divertor target. A summary of the temporal evolution of the outer divertor regimes after type-I ELM events for different outer target recycling regimes is given. As a result of type-I ELMs the upstream density and temperature profiles deplete and are followed by a recovery phase, that reestablishes the pre-ELM upstream parameters. During this recovery phase, in the case of a high recycling divertor prior to the ELM, a sequence of sec-

* Max-Planck-Institut für Plasmaphysik, EURATOM-Association, Boltzmannstr. 2, D-85748, Garching, Germany

Email address: marco.wischmeier@ipp.mpg.de (ASDEX Upgrade team). 
ondary peaks in particle flux not associated with an increased power flux to the target is observed. A possible explanation derived from considerations based on the simple 2-point model is presented, relating the divertor target parameters to the dynamical behavior of the upstream parameters.

\section{Experiment}

The contribution concentrates on two example discharges in ELMy H-mode, one with the outer target being in a low/medium recycling regime (\#17151) and the other being in a high recycling regime (\#19438). Both discharges were lower single null deuterium fuelled plasmas in ASDEX Upgrade with the divertor IIb [3]. Figure 1 shows the poloidal cross section of the magnetic configuration of the two example plasmas. At the time of the discharges most of the plasma facing components of the vessel were covered by tungsten coated tiles with the exception of the LFS limiters and divertor target plates that were composed of CFC. Prior to both experiments, Helium glow cleaning discharges were performed. A time slice with constant $\bar{n}_{e}$ was used for generating coherent ELM averaged data. The inter ELM period of the discharge \#17151 has also been extensively modelled by A.Chankin [4] using the SOLPS5 code package. The principle discharge parameters were a plasma current, $I_{p}=0.8 M A$, toroidal magnetic field, $B_{T}=-2 T$, safety factor, $q_{95}=4.95$ and an initial flat top density of $\bar{n}_{e} \approx 6.0 \times 10^{19} \mathrm{~m}^{-3}$. The additional heating power through Neutral Beam Injection, NBI, was 5.1MW and provided the particle fuelling. The inter ELM peak particle flux to the outer target was $4 \times 10^{22} \mathrm{~m}^{-2} \mathrm{~s}^{-1}$ to $5 \times 10^{22} \mathrm{~m}^{-2} \mathrm{~s}^{-1}$, thus representing a typical low/medium recycling outer divertor target. 
The discharge, \#19438, for comparison had different parameters resulting in pre-ELM particle fluxes being a factor $\sim 2$ higher than in \#17151. Operational values of $I_{p}=1.0 \mathrm{MA}, B_{T}=-2.5 T, q_{95}=4.25$, and a flat top value of $\bar{n}_{e} \approx 7.5 \times 10^{19} \mathrm{~m}^{-3}$ were used in $\# 19438$. The additional heating power was $\sim 7.5 M W$, with $P_{N B I}=5 M W$ and $2.45 M W$ from ICRH heating. In \#19438 the pedestal density, $n_{e}^{\text {ped }}$, was $\sim 6 \times 10^{19} \mathrm{~m}^{-3}$ compared to $\sim 4 \times 10^{19} \mathrm{~m}^{-3}$ in \#17151. The combination of $n_{e}^{p e d}$ and the pre-ELM particle fluxes to the outer target classifies \#19438 as a high density H-mode discharge with a high recycling outer target.

Figure 2 shows the time evolution of the outer divertor $D_{\alpha}$ signal, the stored magnetic energy, $W_{m h d}$, and the line averaged density measured with the horizontal DCN interferometer H-5, $\bar{n}_{e}^{H-5}$. The H-5 line of sight, shown in Fig. 1, makes it more sensitive to changes of the plasma edge density than other interferometer chords. Both discharges have an ELM period of $\sim 10 \mathrm{~ms}-15 \mathrm{~ms}$. During the crash of $W_{m h d}$ and $\bar{n}_{e}^{H-5}$ a peak in the $D_{\alpha}$ signal appears in both discharges. This $D_{\alpha}$ signal is associated to a peak power flux deposited on the outer target plate, measured by an IR camera and an increase in particle flux, target electron temperature, $T_{t}$, and $C I I I$ line radiation. These are typical signatures, related to the power and particles expelled into the SOL by an ELM on a timescale $<1 m s$. The crash in $W_{m h d}$ and $\bar{n}_{e}^{H-5}$ is followed by a continuous recovery up to a critical value and release of the next ELM. The two discharges do not differ in their temporal evolution of the signals for $W_{m h d}$ and $\bar{n}_{e}^{H-5}$ but in the $D_{\alpha}$ signal after an ELM. While \#17151 shows a quiescent recovery phase, the usually observed and published behavior of type-I ELMs, \#19438 has a totally different evolution. The ELM, naming it the "primary" peak, is now followed by a second peak in the $D_{\alpha}$ signal during the recovery phase. This second peak has a much slower rise time and broader temporal 
profile than the "primary" peak. However this is not linked to any depletion of

the $W_{m h d}$ nor $\bar{n}_{e}^{H-5}$ signals. The second peak starts at $\sim 2 m s$ and extends up to $\sim 6 m s$ after the onset of the ELM including strong random fluctuations in its peak value, that can reach the maximum signal strength of the "primary" ELM. Thereafter the intensity drops below pre ELM values before restarting the recovery. A transition to such a double peaked temporal evolution can also be seen when ramping up $\bar{n}_{e}$ in H-mode discharges at constant heating power. In order to reduce the signal noise and base the observations on a large sample of data "coherent averaged" ELMs were produced (for a description of the method see also [5]) and saved in an MDSplus data storage file format. Figure 3 relates the temporal evolution of the $D_{\alpha}$ signal and outer target profiles at three different time slices during the second peak with $t=0 \mathrm{~s}$ being the time of the onset of the ELM. During the second peak of $D_{\alpha}$ the particle flux increases (measured by fixed target Langmuir probes), Fig. 3(c). Neither the peak value nor the profile of the deposited power, Fig.3(b), change and $T_{e}$, Fig. 3(d) remains at low values of $\sim 5 \mathrm{eV}$ until the particle flux has dropped. It then rises up to $10 \mathrm{eV}$ to $20 \mathrm{eV}$. This is consistent with the observation that the second peak is not associated with a drop in $W_{m h d}$ and thus release of power into the SOL and therefore is not a further ELM. The increase in $T_{e}$ is correlated to an increase of the spectroscopic CIII signal confirming the rise of $T_{e}$ at the outer target measured by fixed target Langmuir probes.

\section{Signal interpretation}

The divertor regimes are characterized as follows. After the ELM the divertor switches to cold lower recycling conditions, followed by a cold high recycling 
regime before returning to a hot target at lower recycling. The origin of this second peak in high density discharges is sought. The second peak may be a consequence of an instantaneous recycling of the ions originating from the ELM and reaching the main chamber walls. A large enough influx of recycling neutrals could alter the upstream density profile, an effect being larger in a SOL at high density compared to low density, with the particle source term in the SOL being larger. Structures furthermost away from the separatrix are reached by a radially propagating ELM in less than a $m s$. Recycled molecules would have the lowest thermal speed in the recycling flux. With such thermal speeds the maximum time of flight to the separatrix is $\ll 1 m s$ and a delay of the second peak by $\sim 3-4 m s$ may not be expected due to upstream recycling and re-ionization. A deep penetration of high energy ions into material surfaces may though delay the release of recycled neutrals.

The time scale of the temporal evolution of the second peak is larger than $1 m s$ (larger than the upper limit for the parallel ion sonic transport time of $\sim 450 \mu s[6])$. Therefore any change of the upstream parameters translates into divertor conditions on a time scale $<1 \mathrm{~ms}$. During an ELM upstream and downstream plasma parameters cannot be linked in such a way as the time scale of the event is below the parallel transport time. However, the time scale of the second peak is larger and therefore directly reflects the conditions upstream above the X-point in time. Furthermore the time scale of the peak is $\sim 4 m s$ which is similar to the experimentally measured recovery times for the density profile in the SOL [7]. Note that the recovery time of the SOL density profiles is $\sim 4 m s$, independent of the ELM frequency and may reach up to $\sim 30 \mathrm{~ms}$ for the pedestal. Assuming the second peak to be on average a sequence of transient upstream-downstream equilibria, the upstream conditions during the second peak can be related to the target conditions by 
applying the simple 2-point model [8]. The 2-point model provides a relation between the target particle flux $\Gamma_{t}$ and temperature, $T_{t}$, and the parallel power density $q_{\|}$, upstream density, $n_{u}$, and temperature $T_{u}: \Gamma_{t} \propto n_{u}^{2} q_{\|}^{-3 / 7}$ and $T_{t} \propto$ $q_{\|}^{2} /\left(n_{u}^{2} T_{u}^{2}\right)$. After the ELM $n_{u}$ and $T_{u}$ would be depleted and thus "low" (a consequence of the expulsion of power and particles during the ELM) leading to a cold divertor. The relations can be combined such that:

$$
\frac{\Gamma_{t}}{q_{\|}} \propto \frac{n_{u}^{4 / 7}}{T_{u}^{10 / 7} T_{t}^{5 / 7}}
$$

From experiment it is seen that this ratio increases during the second peak whilst $T_{t}$ changes marginally. Therefore $n_{u}$ must increase faster than $T_{u}$ and the rise of $\Gamma_{t}\left(q_{\|}=\right.$const $)$reflects the recovery time of $\sim 4 m s$ for the upstream density profile. At some point in time $n_{u}$ saturates and $T_{u}$ continues recovering, linked to the energy source being $P_{S O L}$ which may increase due to an increase of $\nabla T_{e}$ inside the separatrix. As a consequence the ratio of $\Gamma_{t} / q_{\|}$decreases and $T_{t}$ increases as is also observed in experiment. The above time dependent relations are shown schematically in Fig.4. To understand the cause of the second peak it shall be noted that the only energy source for the scrape-off layer is the core region and thus any variation of $T_{u}$ depends on $P_{S O L}$. The particle source is a combination of refuelling through the separatrix and ionization sources due to recycling neutrals from plasma facing components. These additional particle sources are usually larger at high density compared to low density and may then lead to a relatively faster recovery of $n_{u}$ compared to $T_{u}$. The additional particle source may originate from a cold divertor more open to neutrals after the ELM or again due to recycling from the MCW. After the second peak at high $T_{t}, \Gamma_{t}$ increases again as a result of the continuous recovery of the upstream profiles increasing the upstream pressure. With the next ELM the cycle then restarts. Direct experimental evidence has not yet been 
reported that the recovery time of the temperature profile may be delayed compared to the density profile at high $\bar{n}_{e}$. Preliminary results from simulations using the SOLPS5.0 fluid Monte Carlo code package [9] as a mock-up tool for ELMs indicate that the upstream density profile does recover faster at high density compared to the upstream temperature profile.

\section{Conclusions}

With increasing $\bar{n}_{e}$ and recycling level at the outer target during the inter ELM phase of type-I ELMs a second temporal peak in particle flux and $D_{\alpha}$ signal is observed at the outer target, which is not related to an increased power flux nor a further release of energy or particles into the SOL upstream. During this second peak the temperature at the target remains low and increases only afterwards. A possible explanation for the second peak at high density has been presented assuming that the time scale of this peak is large compared to parallel sonic transport times such that the target parameters reflect the changes in the upstream density and temperature profiles during the recovery phase after an ELM. The plasma parameters in the divertor are thus linked to the upstream values and the 2-point model can be applied to deduce profile changes upstream. The coherent ELM average of the sequence of secondary peaks in particle flux does indeed have a time scale similar to the recovery time of the upstream density profile in the SOL. It is concluded that at high density the recovery of the density profile may be faster than for the temperature profile, possibly related to additional particle sources in the SOL and changes in $n_{u}$ having a stronger influence on $\Gamma_{t}\left(\propto n_{u}^{2}\right)$. Simulations using the SOLPS5.0 code to simulate the dynamical behavior of the upstream 
profiles are underway and preliminary results indicate such a behavior.

\section{References}

[1] H. Zohm. Plasma Phys. Control. Fusion, 38:105, 1996.

[2] ITER Physics Basis Editors, ITER Physics Expert Group Chairs and Co-Chairs, and ITER Joint Central Team and Physics Integration Unit. Nucl. Fusion, 39:2137-2664, 1999.

[3] R. Neu et al. Plasma Phys. Control. Fusion, 44:825, 2002.

[4] A. Chankin et al. Plasma Phys. Control. Fusion, , 2006. accepted for publication.

[5] R. A. Pitts et al. Nucl. Fusion, 43:1145, 2003.

[6] T. Eich et al. Journal of Nuclear Materials, 337-339:669, 2005.

[7] I. Nunes et al. Nucl. Fusion, 45:1550, 2005.

[8] Keilhacker et al. Plasma Physics and Controlled Nuclear Fusion Research, III:183, 1982. Vienna:IAEA.

[9] R. Schneider et al. Contributions to Plasma Physics, 46(1-2):3, 2006. 


\section{$5 \quad$ Figure captions}

Fig. 1. Poloidal cross section of magnetic configurations of the SNL discharges \#17151 and \#19438 including the line of sight of the DCN interferometer H-5.

Fig. 2. Example time traces of the signals for $W_{m h d}, \bar{n}_{e}^{H-5}$ and divertor $D_{\alpha}$ for \#17151 (a) and \#19438 (b).

Fig. 3. Coherent ELM averaged signals of the $D_{\alpha}$ time trace (a) and the outer target profiles for three distinct time slices during the second $D_{\alpha}$ peak: power deposition profiles from IR (b), particle flux (c) and $T_{e}(\mathrm{~d})$ from LP.

Fig. 4.

Sketch of the time dependence of the outer target particle flow with the upstream density saturating after the recovery time and assuming here for simplicity a constant $q_{\|}$ 


\section{Figures}

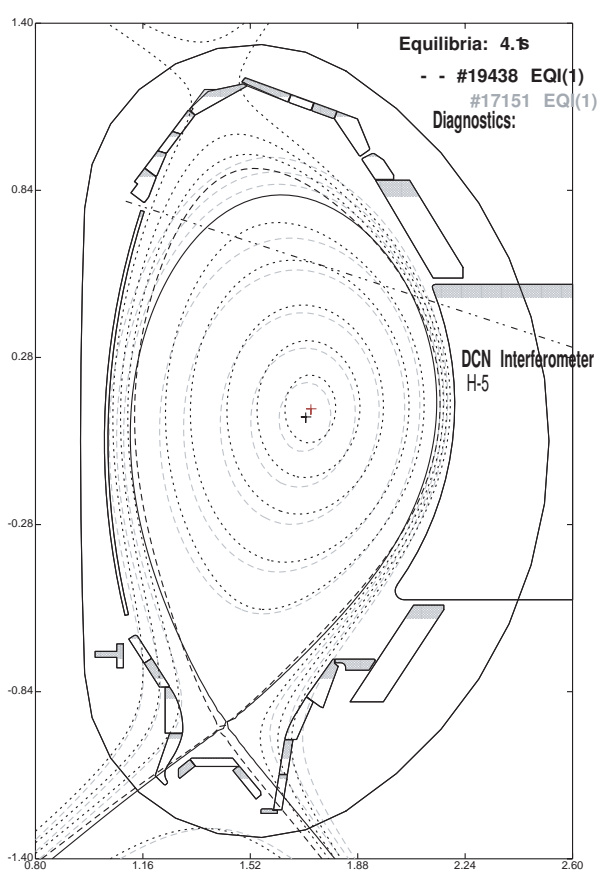

Figure 1
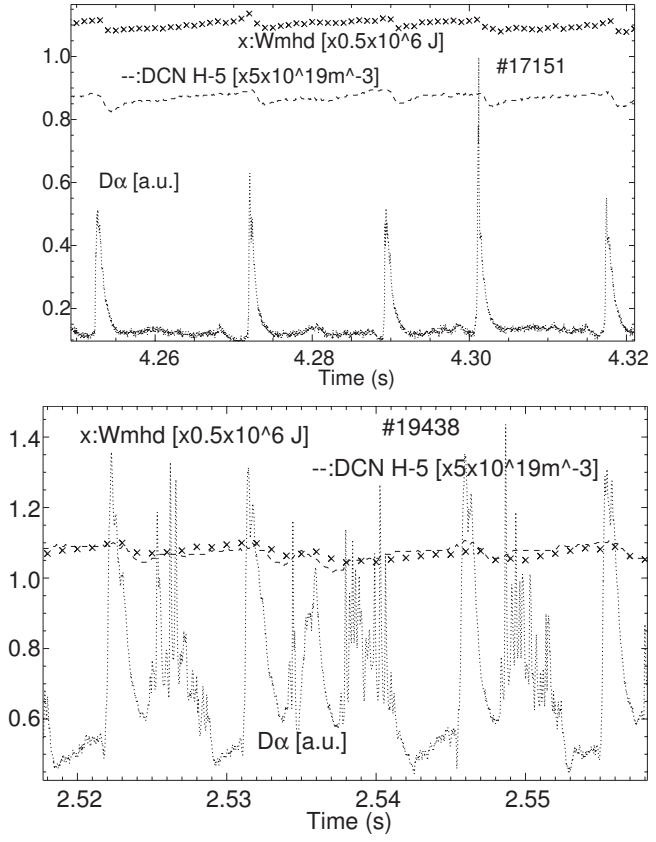

a)

Figure 2 

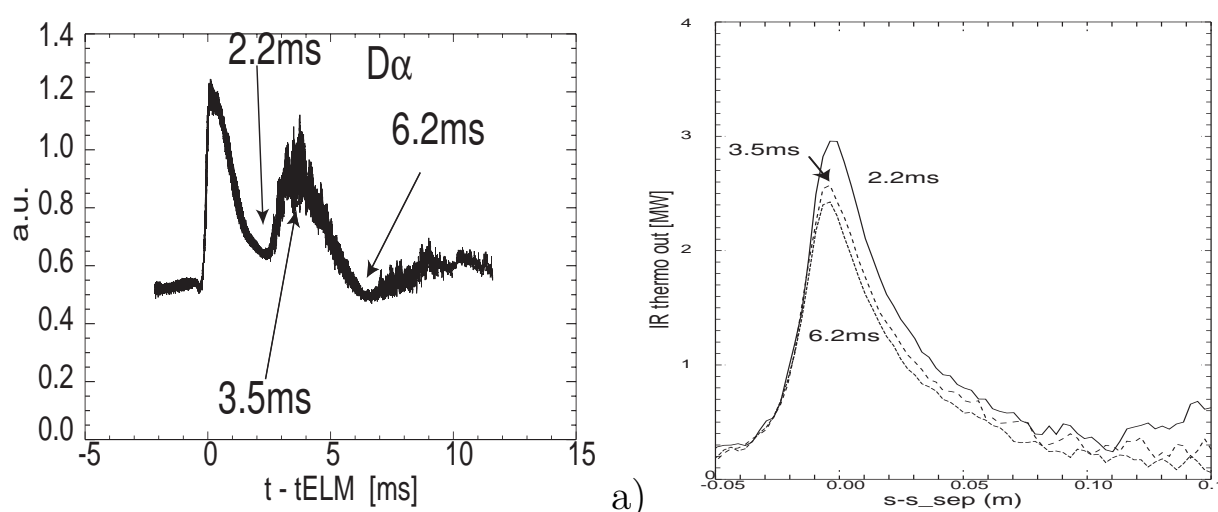

b)
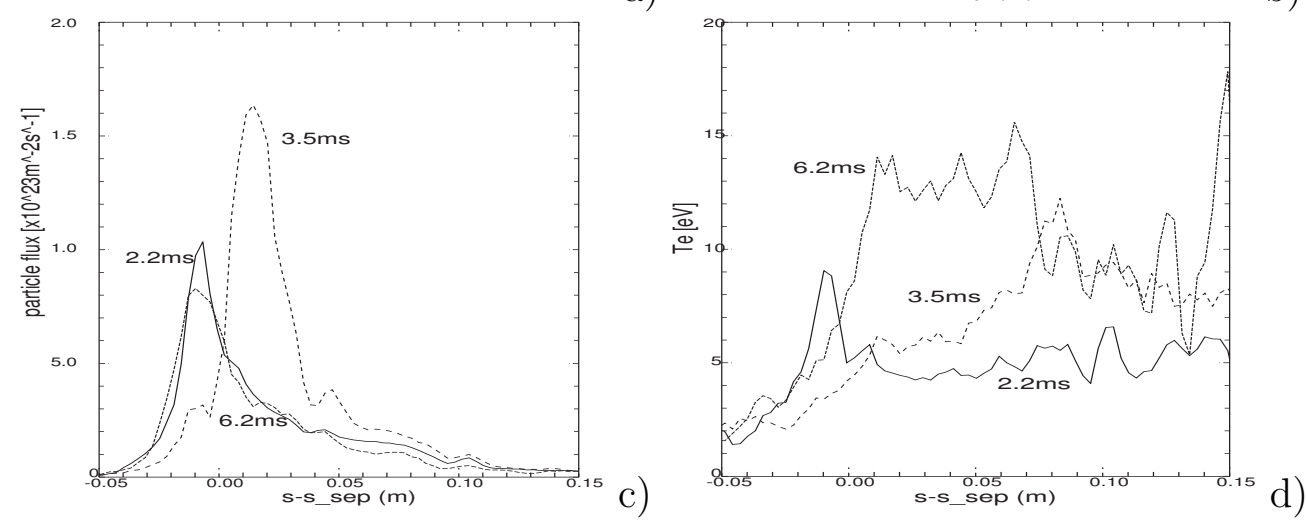

Figure 3

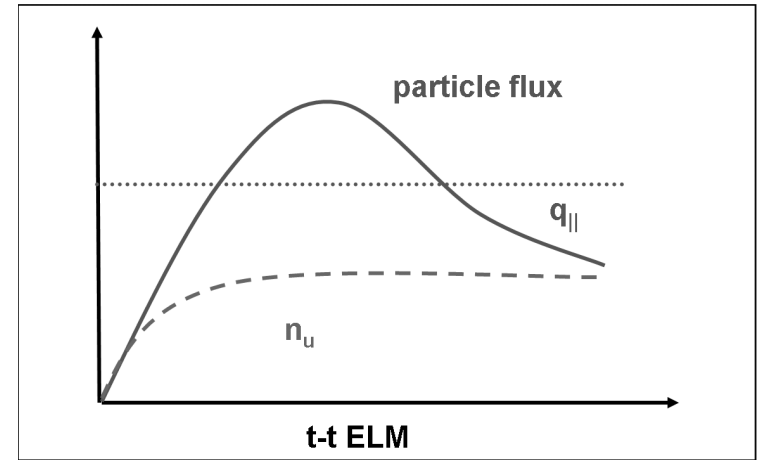

Figure 4 